\title{
INtegration of care for reaching targetS In Diabetic patiEnts: Design of the INSIDE Study
}

\author{
Marco Comaschi (D) - Andrea Di Lenarda - Gerardo Medea • \\ Alberto Aglialoro - Domenico Cucinotta • Michele Gulizia • \\ Giacomo Vespasiani · Guerrino Zuin · Antonio Nicolucci • \\ Federico Spandonaro · Aldo Pietro Maggioni
}

Received: October 8, 2019 / Published online: November 22, 2019

(C) The Author(s) 2019

\section{ABSTRACT}

Introduction: Three Italian scientific associations of different specialties (AMD, Associazione Medici Diabetologi-for diabetologists; ANMCO,Associazione Nazionale Medici Cardiologi Ospedalieri-for cardiologists; SIMG, Società Italiana di Medicina Generale-for General Practitioners) designed this study to assess whether an integrated care organization

Enhanced Digital Features To view enhanced digital features for this article go to: https://doi.org/10.6084/ m9.figshare.10265363.

Electronic Supplementary Material The online version of this article (https://doi.org/10.1007/s13300019-00731-x) contains supplementary material, which is available to authorized users.

\section{Comaschi ( $\square)$}

Internal Medicine Unit, ICLAS GVM Care \&

Research, 16035 Rapallo, Genoa, Italy

e-mail: mcomaschi@gvmnet.it

A. Di Lenarda

Cardiovascular Center, ASS 1, Trieste, Italy

G. Medea

Metabolic Research SIMG, Brescia, Italy

A. Aglialoro

Diabetes Unit ASL 3, Genoa, Italy

D. Cucinotta

Department of Clinical and Experimental Medicine,

University of Messina, Messina, Italy comprising three different specialists can improve adherence and can achieve the guidelines targets in a population of individuals with type 2 diabetes, without established cardiovascular disease but at high risk ( $\geq 20 \%$ at 10 years according to the CUORE.ISS risk cards) compared with the current standards of care provided by the Italian National Health Service.

Methods: Thirty primary care centers (general practitioners, GPs), 30 cardiology centers and 30 diabetes centers have been selected by the scientific associations, disseminated in the national territory, on the basis of proven previous cooperation in other studies. Each primary care center will enroll 100 type 2 diabetic subjects, $>45$ years old, with no established cardiovascular disease, but with a high risk due

\author{
M. Gulizia \\ Cardiology Unit, Garibaldi Nesima Hospital, \\ Catania, Italy \\ G. Vespasiani \\ METEDA Srl, Rome, Italy \\ G. Zuin \\ Cardiology Unit, Mestre Hospital, Venice, Italy \\ A. Nicolucci \\ CORESEARCH, Pescara, Italy \\ F. Spandonaro \\ CREA Sanità, Rome, Italy
}

A. P. Maggioni

ANMCO Center of Research, Florence, Italy 
to the presence of at least one other risk factor besides diabetes over the cutoff [hypertension $>135 / 80 \mathrm{mmHg}$, LDL cholesterol $>70 \mathrm{mg} / \mathrm{dl}$, tobacco smoke, first-degree familiarity for CHD (coronary heart disease), central obesity according the WHO criteria]. Fifteen of 30 selected primary care centers, chosen randomly, will continue the treatment of the 100 identified patients according to their "usual care," driven by Good Clinical Practice and by current guidelines (control group or "UC"-usual care), collecting all available clinical and instrumental data and transferring them to the electronic CRF. The remaining 15, after informed consent, will submit their 100 patients each in a specific integrated pathway, which entails the mandatory operational integration and exchange of information with the diabetes specialists and cardiologists pertaining to the same previously identified area. The integrated care path for the patients in the proband group (IC, integrated care) is based on application of the recommendations of the Italian Guidelines aimed at achieving the proposed targets for the main risk factors $\quad[\mathrm{LDL}<70 \mathrm{mg} / \mathrm{dl} ; \quad \mathrm{SBP}<130 \mathrm{mmHg}$; HbA1c (glycated hemoglobin) $\leq 7 \%$ $(52 \mathrm{mmol} / \mathrm{mol}]$. All the clinical data will be recorded on a shared electronic CRF. The trial will last 3 years: 6 months for the enrollment and randomization of the centers, 6 months for the enrollment of the probands and control subjects, and 2 years of follow-up. The study will be conducted according the Helsinki Declaration on human experimentation ethics.

Planned Outcomes: The primary planned outcome is represented by the increase in the percentage of people that achieve the target values of at least two out of three of the considered risk factors [HbA1c, SBP (systolic blood pressure), LDL cholesterol] compared with the percentage actually achieved in the control group. The secondary outcomes are: (1) a MACE (major adverse cardiac event) composite: non-fatal myocardial infarction, non-fatal stroke, mortality from any cause and hospitalization for cardiovascular disease; (2) the number of early diagnoses of new onset complications; (3) evaluation of adverse events and safety of the probands and control patients; (4) comparative cost analysis and cost-effectiveness analysis.
Keywords: Diabetes; Cardiovascular diseases; Integrated care

\section{Key Summary Points}

Why carry out this study?

The aim of the study is to demonstrate that patient-centered care provided by three different specialists in an integrated way can obtain better outcomes in a population of at-high-risk diabetic patients on a national level.

The hypothesis of the study is that the integration of different competences may allow the actual implementation of the guidelines.

\section{What was learned from the study?}

This is only the design of an ongoing study, and the results are expected within 3 years.

The first thing one can learn from this study, although it is just starting, is the possibility of working together to reach better outcomes.

\section{INTRODUCTION}

In many health systems, integrated care is seen as a possible solution to the growing demand for improved quality of life and health outcomes of multimorbid and long-term care patients. During the last decade, different models and approaches to integrated care have been widely applied and documented across a variety of settings. However, there is no unifying definition or common conceptual understanding of integrated care. Probably the best definition of "integrated care" is the following:

"Integrated health services delivery is defined as an approach to strengthen people-centred health systems through the promotion of the comprehensive delivery of quality services across the life-course, 
designed according to the multidimensional needs of the population and the individual and delivered by a coordinated multidisciplinary team of providers working across settings and levels of care. It should be effectively managed to ensure optimal outcomes and the appropriate use of resources based on the best available evidence, with feedback loops to continuously improve performance and to tackle upstream causes of ill health and to promote well-being through intersectoral and multisectoral actions" [1].

In most cases, primary care in the Italian Health National Service has complex structures (PCCS or UCCP = Unità Complesse di Cure Primarie $=$ Complex Primary Care Units): these are groups of GPs (and sometimes family pediatricians) that work together in the same center in an integrated way with nurses, administrative staff, social workers and medical specialists. PCCS/UCCPs are similar to the Community Health Centers in other parts of the world. However, in the actual organization of the Italian NHS, difficulties in communication and collaboration frequently exist between primary and secondary care settings. According to the Italian Ministry of Health and several academic institutions, it seems that:

1. Integrated care is considered an essential strategy to improve the patient experience of care and health of the population and reduce the cost per capita (triple aim) $[2,3]$.

2. However, there is a lack of published data to support this claim $[4,5]$.

3. Information on integrated care mechanisms is needed to determine the added value of an integrated care strategy within different settings $[4,6,7]$.

The best integrated care systems seem to be those that might implement the chronic care models theorized in the JAMA articles by Bodenheimer and Wagner $[8,9]$. Recently, the European Society of Cardiology in collaboration with the European Association for the Study of Diabetes published their guidelines for the treatment of people with diabetes, prediabetes and ASCVD (atherosclerotic cardiovascular diseases) and summarized the benefit of a "centred care model" in a box [10] (see Table 1, reproduced from the ESC EASD Guidelines 2019).

Type 2 diabetes is perhaps the chronic disease that best fits the requested needs for clinical research on the efficacy of the chronic care model. Several research groups have tried to compare integrated systems versus "usual care" by testing the results on intermediate outcomes, such as HbA1c or blood pressure values. In the USA, Panattoni and collaborators [11] found a significant reduction in $\mathrm{HbA} 1 \mathrm{c}$ at 6 months and a clinically negligible effect on systolic and diastolic blood pressure values. Another interesting experience was made in Boston [12], where a group of elderly people with diabetes was placed in a treatment system that used a pathology register that allowed centralization of all clinical information on a computerized folder and designed a treatment path according to the guidelines. In the groups of patients following this protocol, all the examined parameters (HbA1c, systolic and diastolic pressure, lipids) related to primary outcomes improved significantly. A thorough review, with data coming from different countries, was published on diabetes care about a year ago, and the authors' conclusions are clearly in favor of the chronic care model [13]. However, the outcomes evaluated in the published papers are usually of an intermediate type, and the focus of most of the studies is parameters of metabolic control (HbA1c) and arterial pressure; sometimes, patient satisfaction has also been evaluated. Moreover, these and other published data are nevertheless limited to numerically reduced groups of patients and cannot be translated to large territorial realities, such as those of a national health service. Moreover, almost all the papers published in this field concern quite heterogeneous populations of people with diabetes, without distinction between high or low risk of macrovascular complications. For these reasons, the present study was designed to evaluate a large nationwide sample of subjects with type 2 diabetes, without known cardiovascular disease but at high risk because of the presence of any additional risk factors. 
Table 1 Recommendations for patient-centered care (reproduced with permission from Ref. [10])

\section{Recommendations for patient-centred care of patients with diabetes}

\begin{tabular}{|c|c|c|}
\hline Recommendations & Class $^{\mathrm{a}}$ & Level $^{b}$ \\
\hline $\begin{array}{l}\text { Group-based structured education pro- } \\
\text { grammes are recommended in patients with } \\
\text { DM, to improve DM knowledge, glycaemic } \\
\text { control, disease management, and patient } \\
\text { empowerment. }\end{array}$ & I & A \\
\hline $\begin{array}{l}\text { Patient-centred care is recommended to facili- } \\
\text { tate shared control and decision-making, } \\
\text { within the context of patient priorities and } \\
\text { goals. }\end{array}$ & I & C \\
\hline $\begin{array}{l}\text { Provision of individual empowerment strat- } \\
\text { egies should be considered to enhance self- } \\
\text { efficacy, self-care, and motivation in patients } \\
\text { with DM. }\end{array}$ & Ila & B \\
\hline
\end{tabular}

\section{AIM OF THE STUDY}

The aim was to assess whether an integrated care organization involving three different levels of care is able to produce better adherence and achievement of the guideline targets in a population of people with type 2 diabetes without established cardiovascular disease, but at high risk ( $\geq 20 \%$ at 10 years according to the CUORE.ISS risk cards) [14] compared with the current standards of care provided by the Italian National Health Service.

\section{DESIGN OF THE TRIAL}

The three Scientific Societies (AMD Associazione Medici Diabetologi-Italian College of Diabetologists, ANMCO Associazione Nazionale Cardiologi Ospedalieri-National Association of Hospital Cardiologists, SIMG Società Italiana di Medicina Generale-Italian College of General Practice) promoting the study produced a list of centers disseminated throughout the country with adequate proportionality between the regions. These centers have to guarantee high levels of clinical practice, have experience in clinical research and have documented collaborative capacity with other specialties. In addition to physicians, each primary care unit is staffed with at least one nurse; each diabetes service includes at least three professionals (nurse, dietician, podologist); each cardiology service includes at least two nurses.

From these lists, 30 centers for each of the three specialties are then further selected, with territorial proximity and previous collaborative experience among them, which represent, in their respective locations, the groups of experimenting centers or proband groups (IC, integrated care). The primary care units will have to be characterized by the association of professionals, with a minimum number of ten. The AMD and ANMCO centers will preferably be those that have already collaboratively carried out other joint studies $[15,16]$. The number of centers was chosen in order to perform the study in all the Italian regions. In each center, 
the recording of all the clinical data of the patients belonging to the institution on electronic sheets must be in place, and such data must be exportable on a specific protected web CRF. The primary care units will have to determine from their computer records beforehand the percentage at which their type 2 diabetic patients, presenting the inclusion criteria for the study, reach the targets set by the guidelines (Italian Standards of Diabetes Mellitus Care AMD SID) [17] based on the data available during the previous year. Using their databases, physicians from the 30 selected SIMG centers will extract 100 patients (for each center) with type 2 diabetes and HbA1c ranging between 6.5 and $8.5 \%$, with at least one other major cardiovascular risk factor (arterial hypertension, defined as $\mathrm{BP}>135 / 80 \mathrm{mmHg}$ or in antihypertensive treatment, LDL $>70 \mathrm{mg} / \mathrm{dl}$ or treatment, tobacco smoke, first degree familiarity for $\mathrm{CHD}$, central obesity according to the WHO criteria), but without history, signs or symptoms of previous or ongoing cardiovascular disease, $>45$ years old. Of the 30 selected centers, 15 , chosen randomly by a computer-generated randomization list, will continue the treatment of the 100 patients identified according to their "usual care," driven by Good Clinical Practice and current guidelines (control group or "UC"), collecting all available clinical and instrumental data and transferring them to the CRF. The remaining 15 centers, after receiving informed consent, will involve their 100 patients in a specific integrated pathway (see Appendix 1), which entails the mandatory operational integration and exchange of information with the identified diabetes specialists and cardiologists. The integrated care path for the proband group ("IC") patients is based on the application of the recommendations of the guidelines aimed at achieving the proposed targets for the main risk factors $\quad[\mathrm{LDL}<70 \mathrm{mg} / \mathrm{dl} ; \quad \mathrm{SBP}<130 \mathrm{mmHg}$; $\mathrm{HbA} 1 \mathrm{c} \leq 7 \%(52 \mathrm{mmol} / \mathrm{mol})]$.

\section{Compliance with Ethics Guidelines}

This study will be performed in accordance with the Helsinki Declaration of 1964 and its later amendments and will be submitted to the
Ethics Committee of the Liguria Region at the St Martin Scientific Institute in Genoa, Italy, for approval. All the recruited subjects must receive precise and complete information about the study and have to sign an informed consent to their participation in the study. The consent form for the proband subjects is reported in Appendix 2.

\section{Inclusion Criteria}

1. People with T2DM lasting $>10$ years without overt cardiovascular disease, with HbA1c values between $>7 \% \quad(52 \mathrm{mmol} / \mathrm{mol})$ and $<8.5 \%(69 \mathrm{mmol} / \mathrm{mol})$ and at least one additional risk factor for CVD (see Table 2 from ESC Guidelines 2019) [10].

2. Age $>45$ years.

3. Informed consent.

\section{Exclusion Criteria}

1. Pregnancy;

2. Type 1 diabetes or secondary diabetes;

3. Life expectancy $<1$ year;

4. Psychophysical and social conditions considered not adequate to follow the study protocol by researchers;

5. Chronic kidney disease with eGFR $<45 \mathrm{~m} /$ min (CDK EPI formula).

\section{Planned Outcomes}

The primary planned outcome is an increase in the percentage of patients that achieve the target values of at least two out of three of the considered risk factors (HbA1c, SBP, LDL cholesterol). The secondary outcomes are:

1. MACE (major adverse cardiac events) composite: non-fatal myocardial infarction, non-fatal stroke, mortality from any cause and hospitalization for cardiovascular disease;

2. The number of early diagnoses of new-onset complications;

3. Evaluation of the adverse events and safety of the probands and control patients; 
Table 2 Stratification of risk factors in diabetic patients (reproduced with permission from Ref. [10])

\section{Table 7 Cardiovascular risk categories in patients with diabetes ${ }^{a}$}
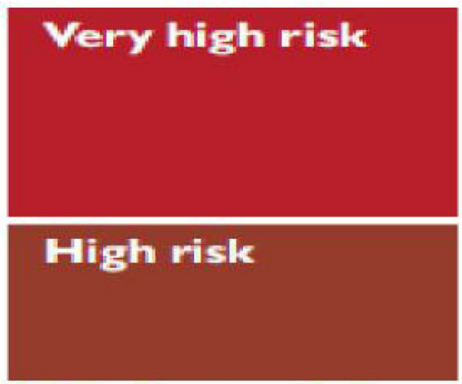

Moderate risk

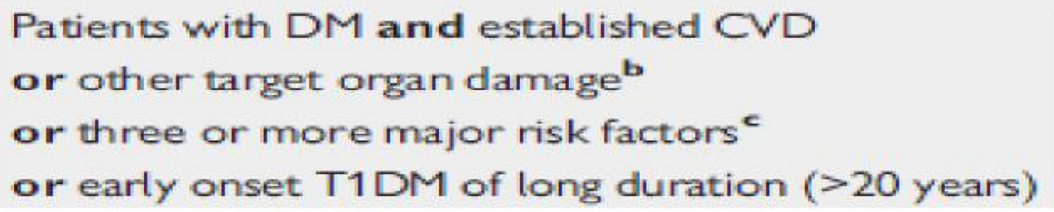

Patients with DM duration $\geq 10$ years without target organ damage plus any other additional risk.

factor

Young patients (T1DM aged $<35$ years or T2DM aged $<50$ years) with DM duration $<10$ years, without other risk factors

\footnotetext{
$C V=$ candiowascular; $C V D=$ cardiowascular disease; $D M=$ diabetes mellitus; T1DM = type 1 diabetes mellitus; T2DM = type 2 diabetes mellitus.

"Modified from the 2016 European Guidelines on cardiovascular disease prevention in clinical practice. ${ }^{27}$

broteinuria, renal impairment defined as eGFR $<30 \mathrm{~mL} / \mathrm{min} / 1.73 \mathrm{~m}^{2}$. left ventricular hypertrophy, or retinopathy.

'Age, hypertension, dyslipidemia, smoking, obesity.
}

4. Comparative cost analysis and cost-effectiveness analysis.

\section{Duration of the Study}

Recruitment of the centers will last 6 months and the enrollment of patients by the centers 1 year; the entire study will be ended in 3 years.

The recruitment starts with the selection of probands from the GP database according to the inclusion criteria (time, 30 days). At time 0 , patients will undergo their first clinical and laboratory controls, and follow-up visits are scheduled at time 180, 360 and 720 days. During this period, the probands will be cared for according to the clinical pathway specified in Appendix 1.

\section{Statistical Considerations}

\section{Sample Size Estimation}

Patients will be enrolled by 30 primary care centers.
The primary end point is represented by the proportion of patients achieving at least two therapeutic targets (HbA1c, systolic blood pressure, LDL cholesterol).

Sample sizes of 1275 in the experimental group and 1275 in the control group, obtained by sampling 15 clusters with 85 subjects each in the experimental group and 15 clusters with 85 subjects each in the control group, achieve $80 \%$ power to detect a difference between the group proportions of 0.05 and a power $>99 \%$ power to detect a difference between the group proportions of 0.10 .

The proportion in the control group is assumed to be 0.15 . The test statistic used is the two-sided $Z$ test (unpooled). The intracluster correlation is 0.005 , and the significance level of the test is 0.05 . Assuming a dropout rate of $15 \%$, a total of 3000 patients will be enrolled (30 clusters of 100 subjects each).

\section{Statistical Analysis}

Descriptive data will be summarized as mean and standard deviation (continuous, normally 
distributed variables), median and interquartile range (continuous, not normally distributed variables) or counts and percentages (categorical variables). Baseline characteristics will be compared using the Student $t$ test, MannWhitney $U$ test or chi-square test, as appropriate. The likelihood to reach the primary end point will be assessed using the chi-square test and binary logistic regression.

Differences between groups and changes during the follow-up within groups for HbA1c, BMI, body weight, blood pressure and lipid parameters will be assessed using mixed models for repeated measurements.

The analysis of the time-dependent secondary end points (i.e., MACE) will be based on a two-sided Cox proportional hazards model.

Incidence rates for major hypoglycemic events will be calculated and expressed as number of events per 100 patient-years with their 95\% CI. Incidence of hypoglycaemic events between groups will be compared using a Poisson regression model with correction for overdispersion.

\section{STRENGTHS AND LIMITATIONS}

This is the first trial aiming to evaluate the efficacy, safety and economic impact of a large system of integrated care for diabetic subjects inside a National Health System. The system is based on a strong relationship among three different health specialists (GPs, cardiologists and diabetologists) and their professional team. If the primary outcome is reached, it will probably be the final evidence that this kind of model is better than the current one.

The performance of this trial presents several risks: for instance, the patient education sessions are intensive for GPs and cardiologists, who already have heavy workloads in daily routine practice. Therefore, the therapeutic education will probably only be done in diabetes centers. In this respect, the work of the nurses, dieticians, podologists and social workers will be crucial. Another problem is the use of several different laboratories for the examination measurements, with no centralization of the samples. However, our choice was determined by the large extension of the participating territories, the need to design a large-scale, pragmatic trial and the very high costs of a centralized system. However, all laboratories have to be certified with a quality system that guarantees the reliability of the results.

The trial will be funded autonomously by the scientific societies, and this fact guarantees the total independence of the researchers.

\section{ACKNOWLEDGEMENTS}

The steering committee thanks the presidents and the directional boards of the three scientific societies, particularly Drs. Claudio Cricelli, Domenico Gabrielli and Domenico Mannino.

Funding. No funding or sponsorship was received for this study protocol or for the publication of this article.

Authorship. All named authors meet the International Committee of Medical Journal Editors (ICMJE) criteria for authorship for this article, take responsibility for the integrity of the work as a whole and have given their approval for this version to be published.

Disclosures. Marco Comaschi, Andrea Di Lenarda, Gerardo Medea, Alberto Aglialoro, Domenico Cucinotta, Michele Gulizia, Giacomo Vespasiani, Guerrino Zuin, Antonio Nicolucci, Federico Spandonaro and Aldo Pietro Maggioni have nothing to disclose.

Compliance with Ethics Guidelines. This study will be performed in accordance with the Helsinki Declaration of 1964 and its later amendments and will be submitted to the Ethics Committee of the Liguria Region at St Martin Scientific Institute in Genoa, Italy, for approval. All the recruited subjects must receive precise and complete information about the study and have to sign an informed consent to participate in the study. The consent form for the proband subjects is reported in Appendix 2. 
Open Access. This article is distributed under the terms of the Creative Commons Attribution-NonCommercial 4.0 International License (http://creativecommons.org/licenses/ by-nc/4.0/), which permits any noncommercial use, distribution, and reproduction in any medium, provided you give appropriate credit to the original author(s) and the source, provide a link to the Creative Commons license, and indicate if changes were made.

\section{REFERENCES}

1. WHO Regional Office for Europe. Strengthening people-centred health systems in the WHO European Region: framework for action on integrated health services delivery. $2016 \mathrm{http} / /$ www.euro. who.int/_data/assets/pdf_file/0004/315787/ 66wd15e_FFA_IHSD_160535.pdf?ua=1. Accessed 20 Oct 2019.

2. Berwick DM. What 'patient-centered' should mean: confessions of an extremist. Health Aff (Millwood). 2009;28(4):w555-65. https://doi.org/10.1377/hlthaff. 28.4.w555.

3. Alderwick H, Ham C. INNOVATION. Look further afield to integrate care locally. Health Serv J. 2015;125(6442):19-21.

4. Valentijn PP, Vrijhoef HJ, Ruwaard D, de Bont A, Arends RY, Bruijnzeels MA. Exploring the success of an integrated primary care partnership: a longitudinal study of collaboration processes. BMC Health Serv Res. 2015;22(15):32.

5. van Dongen SF, Cantekin S, Elemans JA, Rowan AE, Nolte RJ. Functional interlocked systems. Chem Soc Rev. 2014;43(1):99-122.

6. Evans JM, Baker GR, Berta W, Barnsley J. The evolution of integrated health care strategies. Adv Health Care Manag. 2013;15:125-61 (Review).

7. Evans R. MyNHS. Nurs Stand. 2014;29(14):32.

8. Bodenheimer $\mathrm{T}$, Wagner $\mathrm{EH}$, Grumbach $\mathrm{K}$. Improving primary care for patients with chronic illness. JAMA. 2002;288(14):1775-9.

9. Bodenheimer $\mathrm{T}$, Wagner $\mathrm{EH}$, Grumbach $\mathrm{K}$. Improving primary care for patients with chronic illness: the chronic care model, part 2. JAMA. 2002;288(15):1909-14.
10. The Task Force for diabetes, pre-diabetes, and cardiovascular diseases of the European Society of Cardiology (ESC) and the European Association for the Study of Diabetes (EASD)-Francesco Cosentino (ESC Chairperson) (Sweden), Peter J. Grant (EASD Chairperson) (United Kingdom), Victor Aboyans (France), Clifford J. Bailey (United Kingdom), Antonio Ceriello (Italy), Victoria Delgado (Netherlands), Massimo Federici (Italy), Gerasimos Filippatos (Greece), Diederick E. Grobbee (Netherlands), Tina Birgitte Hansen (Denmark), Heikki V. Huikuri (Finland), Isabelle Johansson (Sweden), Peter Jüni (Canada), Maddalena Lettino (Italy), Nikolaus Marx (Germany), Linda G. Mellbin (Sweden), Carl J. Ostgren (Sweden), Bianca Rocca (Italy), Marco Roffi (Switzerland), Naveed Sattar1 (United Kingdom), Petar M. Seferovic (Serbia), Miguel Sousa-Uva (Portugal), Paul Valensi (France), David C. Wheeler (United Kingdom). ESC guidelines on diabetes, prediabetes, and cardiovascular diseases developed in collaboration with the EASD. Eur Heart J. 2019;40(39):3215-7.

11. Panattoni L, Hurlimann L, Wilson C, Durbin M, Tai-Seale M. Workflow standardization of a novel team care model to improve chronic care: a quasiexperimental study. BMC Health Serv Res. 2017;17(1):286.

12. Caruso LB, Clough-Gorr KM, Silliman RA. Improving quality of care for urban older people with diabetes mellitus and cardiovascular disease. J Am Geriatr Soc. 2007;55(10):1656-62 (Epub 2007 Aug 21).

13. Lim LL, Lau ESH, Kong APS, Davies MJ, Levitt NS, Eliasson B, Aguilar-Salinas CA, Ning G, Seino Y, So WY, McGill M, Ogle GD, Orchard TJ, Clarke P, Holman RR, Gregg EW, Gagliardino JJ, Chan JCN. Aspects of multicomponent integrated care promote sustained improvement in surrogate clinical outcomes: a systematic review and meta-analysis. Diabetes Care. 2018;41(6):1312-20. https://doi.org/ $10.2337 /$ dc17-2010.

14. Palmieri L, Panico S, Vanuzzo D, Ferrario M, Pilotto L, Sega R, Cesana G, Giampaoli S, Gruppo di Ricerca del Progetto CUORE. Evaluation of the global cardiovascular absolute risk: the Progetto CUORE individual score. Ann Ist Super Sanita. 2004;40(4): 393-9.

15. Cioffi G, Giorda CB, Chinali M, Di Lenarda A, Faggiano P, Lucci D, Maggioni AP, Masson S, Mureddu GF, Tarantini L, Velussi M, Comaschi M, DYDA Investigators. Analysis of midwall shortening reveals high prevalence of left ventricular myocardial dysfunction in patients with diabetes mellitus: the DYDA study. Eur J Prev Cardiol. 2012;19(5): 935-43. 
16. Giorda CB, Cioffi G, Nada E on behalf of DYDA 2 Study Investigators. Linagliptin and LVD in type 2 diabetic subjects-trial ongoing. Personal communication.
17. Italian Standard of Care for Diabetes Mellitus by AMD and SID 2018. http://aemmedi.it/wp-content/ uploads/2009/06/AMD-Standard-unico1. Accessed 15 Oct 2019. 